\title{
Diagnostic value of transmural perfusion ratio derived from dynamic CT-based myocardial perfusion imaging for the detection of haemodynamically relevant coronary artery stenosis
}

\author{
Adriaan Coenen ${ }^{1,2}$ - Marisa M. Lubbers ${ }^{1,2} \cdot$ Akira Kurata $^{1}$ - Atsushi Kono ${ }^{1}$. \\ Admir Dedic $^{1,2}$ - Raluca G. Chelu ${ }^{1,2}$ - Marcel L. Dijkshoorn ${ }^{1}$. Alexia Rossi ${ }^{1,3}$ • \\ Robert-Jan M. van Geuns ${ }^{1,2} \cdot$ Koen Nieman ${ }^{1,2}$
}

Received: 17 February 2016 /Revised: 9 August 2016 / Accepted: 18 August 2016 / Published online: 4 October 2016

(C) The Author(s) 2016. This article is published with open access at Springerlink.com

\begin{abstract}
Objectives To investigate the additional value of transmural perfusion ratio (TPR) in dynamic CT myocardial perfusion imaging for detection of haemodynamically significant coronary artery disease compared with fractional flow reserve (FFR).

Methods Subjects with suspected or known coronary artery disease were prospectively included and underwent a CT-MPI examination. From the CT-MPI time-point data absolute myocardial blood flow (MBF) values were temporally resolved using a hybrid deconvolution model. An absolute MBF value was measured in the suspected perfusion defect. TPR was defined as the ratio between the subendocardial and subepicardial MBF. TPR and MBF results were compared with invasive FFR using a threshold of 0.80 .

Results Forty-three patients and 94 territories were analysed. The area under the receiver operator curve was larger for MBF (0.78) compared with TPR $(0.65, P=0.026)$. No significant differences were found in diagnostic classification between MBF and TPR with a territory-based accuracy of $77 \%(67-86 \%)$ for
\end{abstract}

Adriaan Coenen

a.coenen@erasmusmc.nl

Department of Radiology, Erasmus University Medical Center, Rotterdam, the Netherlands

2 Department of Cardiology, Erasmus University Medical Center, Rotterdam, the Netherlands

3 NIHR Cardiovascular Biomedical Research Unit at Barts, William Harvey Research Institute, Barts and The London School of Medicine and Dentistry, Queen Mary University of London \& Department of Cardiology, Barts Health NHS Trust, London, UK
MBF compared with $70 \%(60-81 \%)$ for TPR. Combined MBF and TPR classification did not improve the diagnostic classification.

Conclusions Dynamic CT-MPI-based transmural perfusion ratio predicts haemodynamically significant coronary artery disease. However, diagnostic performance of dynamic CT-MPIderived TPR is inferior to quantified MBF and has limited incremental value.

Key Points

- The transmural perfusion ratio from dynamic CT-MPI predicts functional obstructive coronary artery disease

- Performance of the transmural perfusion ratio is inferior to quantified myocardial blood flow

- The incremental value of the transmural perfusion ratio is limited

Keywords Coronary artery disease $\cdot$ Perfusion $\cdot$ Fractional flow reserve, myocardial · Tomography $\cdot \mathrm{X}$-ray computed

$\begin{array}{ll}\text { Abbreviations } \\ \text { AUC } & \text { area under the ROC curve } \\ \text { CAD } & \text { coronary artery disease } \\ \text { CT-MPI } & \text { computed tomography-myocardial } \\ & \text { perfusion imaging } \\ \text { FFR } & \text { fraction flow reserve } \\ \text { MBF } & \text { myocardial blood flow } \\ \text { SPECT } & \text { single-photon emission computed tomography } \\ \text { ROI } & \text { region of interest } \\ \text { TAC } & \text { time-attenuation curve } \\ \text { TPR } & \text { transmural perfusion ratio }\end{array}$




\section{Introduction}

Dynamic computed tomography myocardial perfusion imaging (CT-MPI) is based on sequential scanning of the myocardium during the first pass of a contrast bolus. The dynamic imaging of the contrast medium allows for a non-invasive quantification of myocardial blood flow (MBF), until now mainly performed with either magnetic resonance imaging or positron emission tomography $[1,2]$. With recent developments in CT scanners this technique also became available for CT imaging [3, 4]. The diagnostic performance of CT-MPI compared with fractional flow reserve is good [5-7]. However, possible underestimation of absolute MBF values by CT-MPI is a potential concern $[4,8]$.

Reduced myocardial perfusion due to coronary artery disease (CAD) tends to be more pronounced in the subendocardium [9]. The high spatial resolution of CT allows for distinguishing the subendocardium and subepicardium. A method to utilise the susceptibility of the subendocardium for ischaemia is the transmural perfusion ratio (TPR) [10]. TPR is the ratio between subendocardium and subepicardium perfusion. As TPR is a relative index we hypothesised it would be less influenced by lower absolute MBF values and improve the diagnostic performance of CT-MPI.

In this study TPR and MBF based on dynamic CT-MPI are investigated individually and in combination, and compared with the invasive fractional flow reserve (FFR).

\section{Methods}

\section{Study design}

The local institutional review board approved this prospective study. Written informed consent was obtained from all patients. This study included cases from a previous study investigating the diagnostic performance of CT-MPI [7]. Patients with suspected or known CAD referred for invasive angiography were prospectively recruited. Included patients underwent a dynamic CT-MPI examination 1-14 days before invasive angiography.

This study was designed to investigate the ability of CTMPI to detect ischaemia; therefore only territories with an FFR measurement in the associated coronary artery were included. Territories associated with a (sub)total occluded coronary artery where no FFR measurement could be performed were not included in the analysis.

\section{Recruitment and population}

Patients with suspected or known coronary artery disease referred for invasive angiography were recruited in the time period December 2010 until December 2014. Exclusion criteria were younger than 40 years old, impaired renal function (serum creatinine $>120 \mu \mathrm{mol} / \mathrm{l}$ ), possible pregnancy or breast feeding, body weight over $120 \mathrm{~kg}$, use of clopidogrel, contra-indications for iodine contrast medium, or contraindications for adenosine.

\section{CT-MPI acquisition}

All patients were requested to refrain from caffeine intake $24 \mathrm{~h}$ prior to the examination. In both arms 18-gauge cannulas were inserted in the antecubital veins. Blood pressure and ECG were monitored during the examination. Forty patients were scanned with a second-generation dual-source CT scanner and three patients with a third-generation dual-source CT scanner (SOMATOM Definition Flash and SOMATOM Force, Siemens Medical Solutions, Forchheim, Germany). Adenosine was infused at a rate of $140 \mu \mathrm{g} / \mathrm{kg} / \mathrm{min}$. CT-MPI acquisition was started $3 \mathrm{~min}$ after start of adenosine infusion.

The acquisition protocol consisted of coronary CT angiography, a non-contrast scan and the dynamic CT-MPI scan. The non-contrast scan was acquired during end systole and served for planning of the CT-MPI. Before the CT-MPI acquisition all patients received sublingual nitroglycerine. Intravenous beta blockers were used in patients with high heart rates prior to the coronary $\mathrm{CT}$ angiography, but very infrequently $(N=3)$ as these potentially affect the CT-MPI performance. After $3 \mathrm{~min}$ of adenosine infusion, $50 \mathrm{ml}$ of contrast medium (Ultravist, $370 \mathrm{mgI} / \mathrm{ml}$; Bayer, Berlin, Germany) was injected at $6 \mathrm{ml} / \mathrm{s}$, followed by a saline bolus of $40 \mathrm{ml}$. All CT-MPI studies were made with an axial scan mode at $250 \mathrm{~ms}$ after the $\mathrm{R}$ wave (end systolic). To sufficiently cover the left ventricle the myocardial acquisition was performed in alternating cranial and caudal table positions (shuttle mode), acquiring two slightly overlapping data sets [3]. CT-MPI acquisition was started $5 \mathrm{~s}$ after the start of the contrast medium injection. Patients were asked to hold their breath during the entire dynamic CT-MPI acquisition (30-35 s). The number of time points acquired varied per patient depending on the heart rate: 1 patient had 9 cranial and caudal time points, 11 patients had 10, 6 patients had 11,12 patients had 12, 9 patients had 13, and 4 patients had 14 time points.

The second-generation dual-source CT scanner used the following scan parameters: collimation $2 \times 64 \times 0.6-\mathrm{mm}$ detector collimation with flying z-spot technique [11], gantry rotation time $280 \mathrm{~ms}$, temporal resolution $75 \mathrm{~ms}$, tube voltage/current $100 \mathrm{kVp} / 300 \mathrm{mAs}$ and shuttle-mode coverage $73 \mathrm{~mm}$.

The third-generation dual-source CT scanner used the following scan parameters: collimation $2 \times 96 \times 0.6-\mathrm{mm}$ detector collimation with flying $z$-spot technique, gantry rotation time $250 \mathrm{~ms}$ and temporal resolution $66 \mathrm{~ms}$; Care-Kv [12] was used with reference settings for tube voltage/current: $80 \mathrm{kVp} /$ $300 \mathrm{mAs}$ and shuttle-mode coverage $102 \mathrm{~mm}$. 


\section{Post processing}

The CT-MPI images were reconstructed using a dedicated kernel for reduction of iodine beam-hardening artefacts (b23f, Qr36) and transferred to a CT-MPI analysis software package (Volume Perfusion CT body, Syngo Somaris/7; Siemens, Germany). Motion correction was applied if necessary to correct for breathing displacement. The motion correction algorithm uses a time point selected by the user (with contrast in the left and right ventricle and smooth connection between the cranial and caudal section) and then registers the other time points to the selected time point using non-rigid registration. The left ventricle is segmented by combining thresholding and peak enhancement [13]. The change of attenuation in the myocardium over time was computed by creating time-attenuation curves (TACs). For quantification of the MBF the influx of contrast bolus was measured with an arterial input function (AIF). The AIF was measured by placement of an ROI in the descending aorta in the CT-MPI images. Precision of the AIF was increased by including both the cranial and caudal sections (double sampling). For quantification of the MBF the myocardial TACs were coupled with the AIF using a hybrid deconvolution model. The model generates a perfusion model curve based on the change in attenuation using a simplified impulse residue function for modelling the interaction between the intra- and extracellular compartments. The MBF was computed on a per voxel basis by dividing the maximal slope of the model curve for the myocardial tissue by the maximum AIF [3, 4, 14]. MBF data sets were reconstructed with a $512 \times 512$ matrix resulting in a pixel size of $0.35 \times 0.35 \mathrm{~mm}$ and were reconstructed as a stack of colour-coded maps with a slice thickness of $3 \mathrm{~mm}$ and an increment of $1.5 \mathrm{~mm}$.

\section{Image analysis}

MBF and TPR were individually evaluated by readers with previous experience in dynamic CT-MPI examinations. Both readers were provided with the colour-coded CT-MPI data sets. For each patient a list of vessels investigated by FFR was provided. To ensure correct territory-vessel correspondence left or right coronary dominance was provided for each patient. All readers were asked to measure MBF or TPR value corresponding to the vessel where the FFR measurements were performed. Each independent reader was blinded to all other medical information.

Within the MBF short axis slice interpreted as representing the myocardium dependent on the vessel in which the FFR was made, a freehand ROI was placed surrounding the suspected perfusion defect (Syngo Via 2.0, Siemens AG, Germany). The freehand ROI had a minimal area of $50 \mathrm{~mm}^{2}$. Careful considerations were made to prevent inclusion of artefacts in the ROI.

For TPR the CT-MPI colour-coded maps were visually assessed to identify the slice most representative for a subendocardial/subepicardial ratio. The section of interest was loaded onto a dedicated image analysis application (ImageJ 1.48, National Institutes of Health, USA) [15]. To measure the transmural differences in the MBF a series of linear samples perpendicular to the myocardial surface was taken at 0.4-mm equal intervals (Fig. 1). The mean MBF values from the pixels under the line are projected in the transmural MBF profile curve. Care was taken not to sample too close to the LV lumen and epicardial border as the MBF absolute values are unreliable because of displacement artefacts. From the short-axis MBF image and the MBF profile curve the user selected the endocardial and epicardial
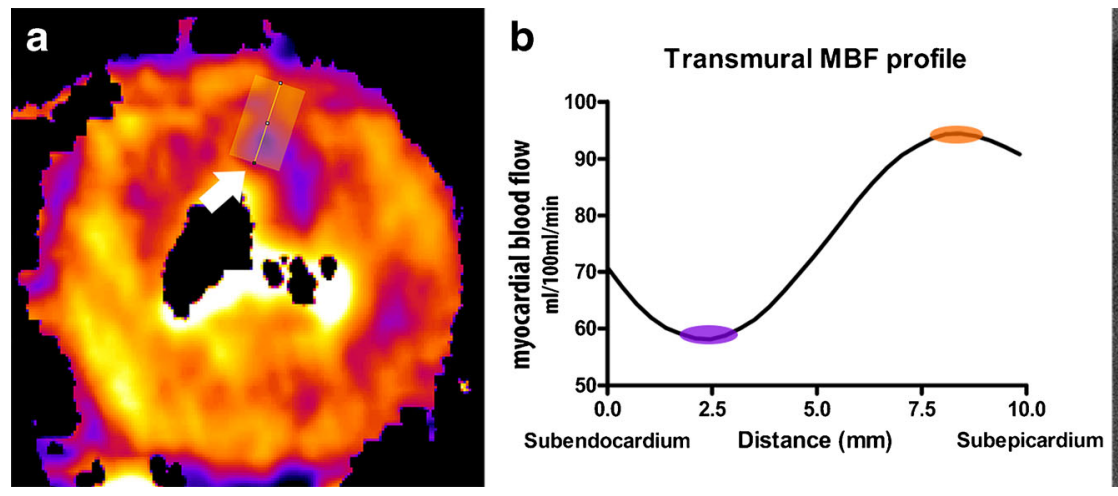

Fig. 1 TPR case example: 65-year-old male presenting after exertional collapse. a Short-axis CT-MPI image with the transmural perfusion line placed in the anterior-lateral segment (white arrow). b The transmural MBF profile. The subendocardial MBF was $58 \mathrm{ml} / 100 \mathrm{ml} / \mathrm{min}$ (purple marker) and $91 \mathrm{ml} / 100 \mathrm{ml} / \mathrm{min}$ in the subepicardium (orange marker). The TPR was 0.64 (58/91) and thus considered positive for ischaemia. c Invasive angiography showing a stenosis in the proximal LAD with an
FFR of 0.69. A subtotal stenosis was directly stented in the LCX (arrowhead); as such no FFR measurement was performed. In panel a however a perfusion defect with a transmural perfusion ratio can be seen in the territory associated with the LCX. The RCA was normal with an FFR of 0.91. RCA: right coronary artery, $L A D$ : left anterior descending artery, $L C X$ : left circumflex artery, $T P R$ : transmural perfusion ratio, $M B F$ : myocardial blood flow, FFR: fractional flow reserve 
positions. The TPR was calculated by dividing the subendocardial by the subepicardial MBF.

\section{Invasive angiography and fractional flow reserve}

Invasive coronary angiography was performed according to local clinical standards. Prior to the invasive angiography intracoronary nitroglycerine was given, as is the standard in our centre. Invasive FFR was performed in all vessels with a visual stenosis grade between $30-90 \%$ by invasive angiography. By protocol, an FFR pressure wire (PressureWire Aeris/ Certus, St. Jude Medical, St. Paul, USA, or Prime/Combo Wire, Volcano, San Diego, CA, USA) was placed distal to the stenosis of interest, after which hyperaemia was induced by intravenous infusion of adenosine at $140 \mu \mathrm{g} / \mathrm{kg} / \mathrm{min}$. An invasive FFR $\leq 0.80$ was considered haemodynamically significant.

\section{Statistics}

Absolute variables are represented as total and percentage, continuous variables as mean and standard deviation $( \pm)$. The mean values for MBF and TPR for normal and ischaemic territories were compared with an unpaired two-sided independent $t$-test. Pearson coefficient correlation was calculated for respectively MBF and TPR against invasive FFR. The receiver-operator characteristic (ROC) curves including the area under the curve (AUC) were presented for MBF and TPR. To investigate the combined diagnostic performance for MBF and TPR an ROC curve was also plotted for a new combined variable MBF multiplied by TPR $(\mathrm{MBF} \times \mathrm{TPR})$. The optimal threshold for MBF and TPR diagnostic accuracy was calculated using the Youden index [16]. A sub-analysis was made for territories with an intermediate MBF between $50-100 \mathrm{ml} / 100 \mathrm{ml} / \mathrm{min}$, as these represent territories with MBF values close to the diagnostic threshold [5, 7]. Diagnostic performance was evaluated as sensitivity, specificity, positive predictive value, negative predictive value and accuracy, with their corresponding $95 \%$ confidence intervals (CI). The $95 \%$ confidence intervals were corrected for withinsubject clustering of data using variance adjustment [17]. MBF and TPR were displayed against each other with territories classified as normal or ischaemic. Inter-observer variability was determined for $72(75 \%)$ randomly selected territories by intraclass correlation coefficient for absolute MBF and TPR; diagnostic classification was compared using kappa statistics. Results were reported on a per-territory and per-patient basis and in accordance with the STARD initiative (Standard for Reporting Diagnostic accuracy) [18]. Most statistical analyses were made using SPSS (version 21, IBM Corp., Armonk NY, USA), while MedCalc (version 13.0; MedCalc Software, Ostend, Belgium) was used to compare the AUCs by using the method of DeLong et al. [19].

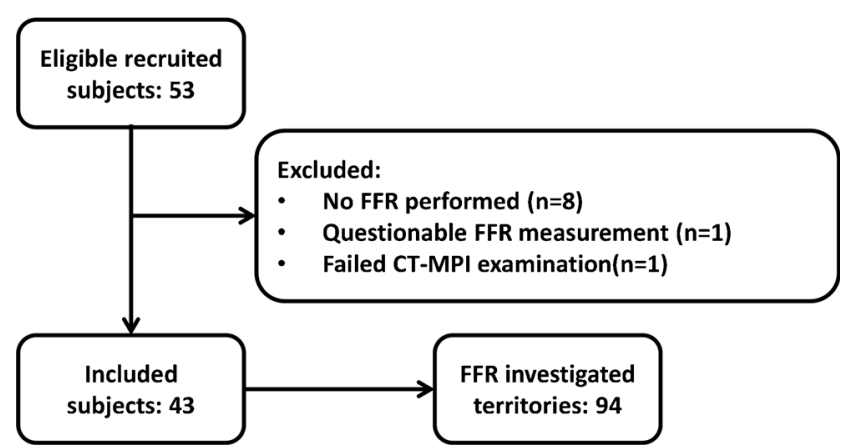

Fig. 2 Inclusion flow chart

\section{Results}

Fifty-three patients were recruited; 10 patients were excluded, 8 because of a lack of invasive FFR measurements (Fig. 2), resulting in a study population consisting of 43 patients, in whom 94 vessels were analysed by invasive FFR (Table 1). The mean FFR was $0.79 \pm 0.17$, with 48 vessels being considered haemodynamically significant with an FFR $\leq 0.80$. The mean doselength product for the CT-MPI acquisition was $640 \pm$ $135 \mathrm{mGy}-\mathrm{cm}$, resulting in an effective dose of $9.0 \pm 1.9 \mathrm{mSv}$ applying a conversion factor of 0.014 (Fig. 1).

The mean MBF for FFR confirmed ischaemic territories was $71.3 \pm 24.3 \mathrm{ml} / 100 \mathrm{ml} / \mathrm{min}$ and for normal territories $92.2 \pm 21.6 \mathrm{ml} / 100 \mathrm{ml} / \mathrm{min}$ (Fig. 3). The Pearson correlation

Table 1 Patient characteristics

\begin{tabular}{lc}
\hline Number of patients, $n$ & 43 \\
\hline Age (years) & $62.6 \pm 8.7$ \\
Male gender, $n(\%)$ & $36(84)$ \\
Body mass index $\left(\mathrm{kg} / \mathrm{m}^{2}\right) *$ & $20.1 \pm 2.3$ \\
Body surface area $\left(\mathrm{m}^{2}\right) *$ & $2.0 \pm 0.14$ \\
Cardiovascular risk factors, $n(\%)$ & \\
Hypertension & $27(63)$ \\
Dyslipidaemia & $20(47)$ \\
Diabetes & $7(16)$ \\
Family history of CAD & $17(40)$ \\
Smoking within the last year & $10(22)$ \\
Prior myocardial infarction, $n(\%) \dagger$ & $8(19)$ \\
Prior PCI, $n(\%) \dagger$ & $5(12)$ \\
Agatston coronary calcium score $\dagger$ & $628(265-1450)$ \\
Heart rate during rest & $63.4 \pm 12.9$ \\
Heart rate during hyperaemic CT-MPI. & $83.0 \pm 13.7$ \\
\hline
\end{tabular}

Values are reported as mean and \pm standard deviation or absolute number $n$ and percentage (\%). CAD, coronary artery disease; PCI, percutaneous coronary intervention

*In four patients length and weight data were not available $\dagger$ Not in the vessel territories interrogated by invasive FFR $\ddagger$ Represented in median and (quartiles) 


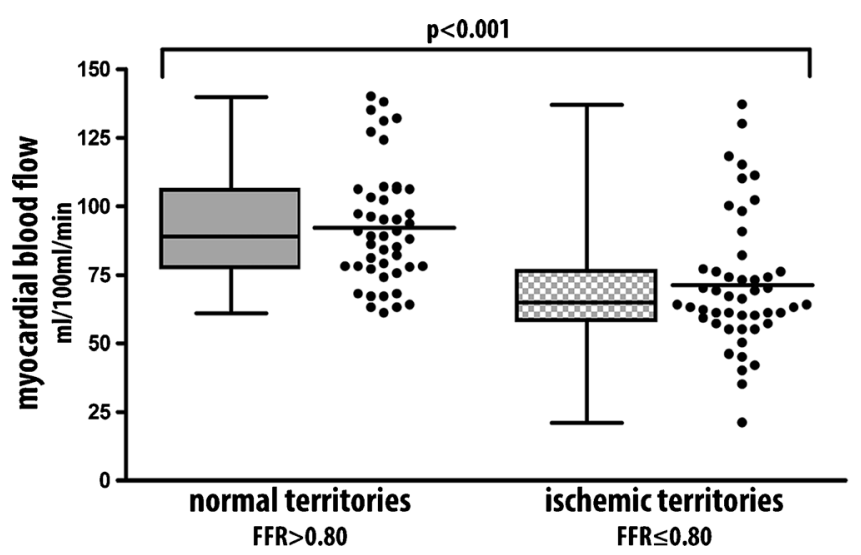

Fig. $3 \mathrm{MBF}$ and TPR: Median and mean myocardial blood flow and transmural perfusion ratio in 94 territories for normal $(N=46)$ and ischaemic $(N=48)$ territories. Normal territory defined as invasive

coefficient was 0.55 for MBF directly compared with invasive FFR. The area under the curve (AUC) was 0.78 (Fig. 4). Optimal threshold for diagnostic classification was $\leq 76 \mathrm{ml} /$ $100 \mathrm{ml} / \mathrm{min}$. The territory-based accuracy for MBF was $77 \%(67-86 \%)$ (Table 2).

The mean TPR for ischaemic territories was $0.85 \pm 0.31$ (Fig. 3). Pearson correlation between TPR and invasive FFR was 0.37 . The AUC for TPR was 0.65 and significantly smaller than for $\mathrm{MBF}(P=0.026)$. The optimal threshold for diagnostic classification was $\leq 0.82$ (Fig. 4). The territory-based accuracy of TPR was $70 \%(60-81 \%)$ (Table 2).

The AUC for the MBF and TRP combined was 0.71 significantly higher than for TPR alone $(P=0.032)$, and the difference with MBF just failed to reach statistical significance $(P=0.070)$. To further investigate the incremental value of TPR a combined

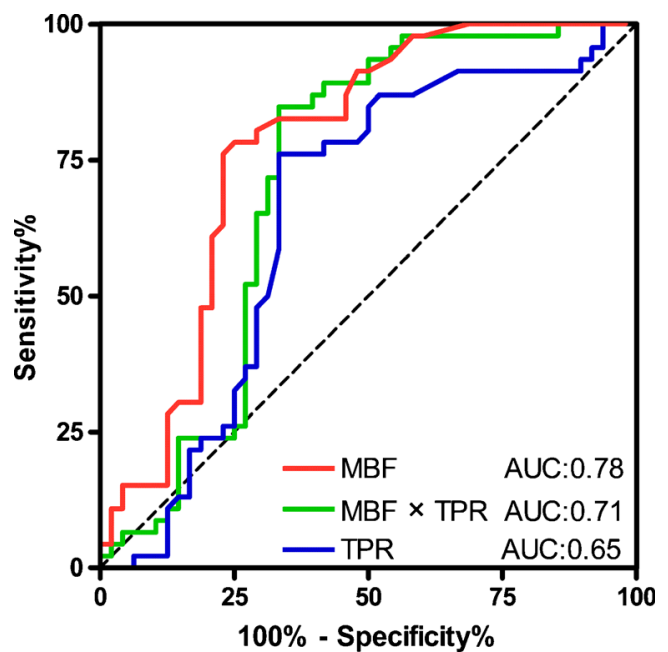

Fig. 4 ROC: Receiver-operator curves for MBF and TPR validated against FFR using a threshold of 0.80 for haemodynamic significance. Area under the curve for MBF was 0.78 (95\% CI: 0.67-0.87), for TPR 0.65 (95\% CI: 0.53-0.77) and for MBF $\times$ TPR 0.71 (95\% CI: 0.60-0.82). The optimal diagnostic threshold was calculated at $76 \mathrm{ml} / 100 \mathrm{ml} / \mathrm{min}$ for MBF and 0.82 for TPR. $M B F$ : myocardial blood flow, TPR: transmural perfusion ratio, FFR: fractional flow reserve, $C I$ : confidence interval

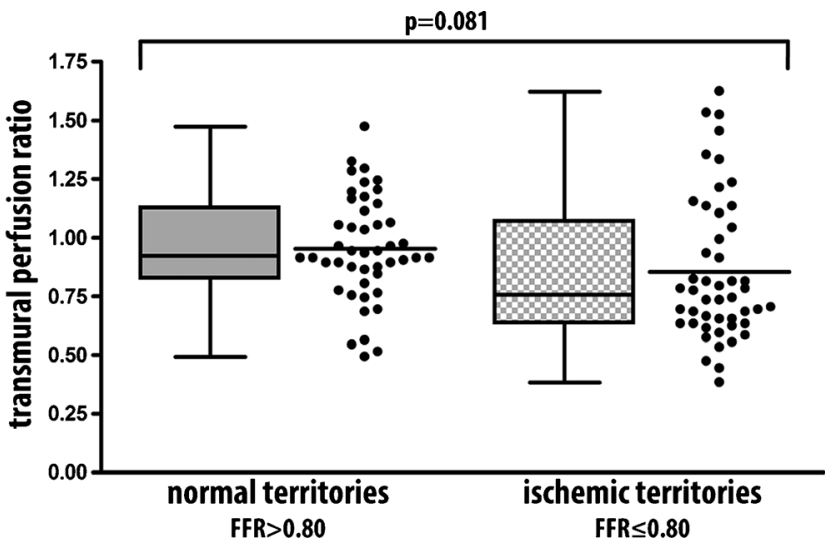

FFR $>0.80$, and ischaemic territories as FFR $\leq 0.80$. FFR: fractional flow reserve

interpretation is shown in Fig. 5. Concordance between the MBF and TPR diagnostic classification was present in the majority of the territories $(74 \%)$. For territories with concordant abnormal MBF and TPR a trend towards an increased positive predictive value was observed. A combined classification did not yield significant improvement in diagnostic accuracy, not for all territories or for the territories with an intermediate MBF between 50$100 \mathrm{ml} / 100 \mathrm{ml} / \mathrm{min}$ (Table 2).

The inter-observer variability for TPR was moderate to good with an intra-class correlation coefficient of 0.77 and a kappa of 0.66 . For MBF reproducibility was better with an intra-class correlation of 0.84 and a kappa of 0.77 .

Only three patients were scanned with a third-generation DSCT. Reanalysis after exclusion of these cases did not affect the results (data not shown).

\section{Discussion}

The main findings of this study are (1) the transmural perfusion ratio from dynamic CT-MPI predicts functionally flowlimiting $\mathrm{CAD}$; (2) the transmural perfusion ratio based on dynamic CT-MPI myocardial blood flow maps is inferior to quantified myocardial blood flow.

The subendocardial layer is more susceptible for ischaemia, which is thought to be due to a reduction in the diastolic perfusion time interval, higher contractile intra-myocardial tissue pressures and differences in coronary microvasculature $[9,20]$. By comparing the subendocardial and subepicardial perfusion the susceptibility of the endocardium for ischaemia can be used as a diagnostic criteria.

Barmeyer et al. found that subendocardial/subepicardial ratio using stress MRI perfusion was associated with functional CAD in comparison to the coronary flow reserve; however measurements taken only in the subendocardial layer showed superior diagnostic performance [21]. Using oxygen positron emission tomography MPI a similar association between TPR 
Table 2 Diagnostic performance

\begin{tabular}{lcccclllll}
\hline All vessels $(n=94)$ & TP & FP & TN & FN & Sensitivity & Specificity & PPV & NPV & Accuracy \\
\hline MBF & 36 & 10 & 36 & 12 & $75 \%(62-88 \%)$ & $78 \%(66-90 \%)$ & $78 \%(65-91 \%)$ & $75 \%(63-87 \%)$ & $77 \%(67-86 \%)$ \\
TPR & 31 & 11 & 35 & 17 & $65 \%(50-79 \%)$ & $76 \%(64-89 \%)$ & $74 \%(59-88 \%)$ & $67 \%(54-80 \%)$ & $70 \%(60-81 \%)$ \\
MBF 50-100 $(n=65)$ & & & & & & & & $78 \%(71-98 \%)$ & $78 \%(68-90 \%)$ \\
MBF & 29 & 10 & 22 & 4 & $88 \%(76-100 \%)$ & $69 \%(53-85 \%)$ & $74 \%(60-89 \%)$ & $85 \%(59 \%)$ \\
TPR & 24 & 8 & 24 & 9 & $73 \%(54-89 \%)$ & $75 \%(60-90 \%)$ & $75 \%(59-91 \%)$ & $73 \%(57-88 \%)$ & $74 \%(62-86 \%)$ \\
\hline
\end{tabular}

Diagnostic performance with invasive FFR using a threshold of $\leq 0.80$. Territories with an MBF $\leq 76 \mathrm{ml} / 100 \mathrm{ml} / \mathrm{min}$ and TPR $\leq 0.82$ were considered positive for ischaemia. A sub-analysis is made for territories with an intermediate MBF between $50 \mathrm{and} 100 \mathrm{ml} / 100 \mathrm{ml} / \mathrm{min}$. FFR: fractional flow reserve, MBF, myocardial blood flow, TPR: transmural perfusion ratio, PPV: positive predictive value, NPV: negative predictive value

and functional stenosis measurement was found; however similar to our study TPR was inferior to quantified myocardial perfusion measurements [22]. The high spatial resolution of $\mathrm{CT}$ is well suited for differentiating the myocardial layers and identification of subendocardial perfusion differences. George et al. showed the potential of the transmural perfusion ratio using static CT-MPI to detect ischaemia, validated by a combination of quantitative angiography analysis and SPECT [10]. In another static CT-MPI study validated by SPECT good diagnostic performance of a transmural perfusion gradient was found [23]. Ko et al. found static rest and stress CTMPI assets visually were of incremental value to coronary CT angiography [24]. More recently Yang et al. published visual static CT-MPI assessment performed better than the transmural perfusion ratio, validated by FFR [25]. In these studies a segmental-based TPR was calculated while for the epicardial layer the entire circumferential attenuation was averaged. In our study we used the epicardial myocardial blood flow at the location of the suspected perfusion defect. Because calculated MBF values vary between different regions of the heart, even in the absence of CAD, we compared the subendocardial MBF values against the adjutant subepicardial layer.
Several studies showed good diagnostic performance of dynamic CT-MPI to identify haemodynamically significant coronary artery disease compared with the fractional flow reserve $[5-7,26]$. A potential concern is the relatively low absolute myocardial blood flow values computed with dynamic CT-MPI [4, 27, 28]. We hypothesised that a relative endocardial/epicardial perfusion ratio would be less vulnerable to individual variations in global MBF values and would be more sensitive in the identification of subtle perfusion defects.

This study shows that the transmural perfusion ratio identifies haemodynamically relevant coronary artery disease. However, no significant incremental value of TPR on top of MBF was found. In patients with an abnormal MBF, addition of TPR could reclassify a number of false-positive results; however a statistically significant improvement could not be demonstrated in this modestly sized cohort. There are several possible explanations for the negative outcome in this study: The TPR methodology in this study is different from methods previously used in static CT-MPI. In dynamic CT-MPI the endocardial zone directly adjacent to the left ventricle cavity is prone to artefacts related to myocardial displacement, beam

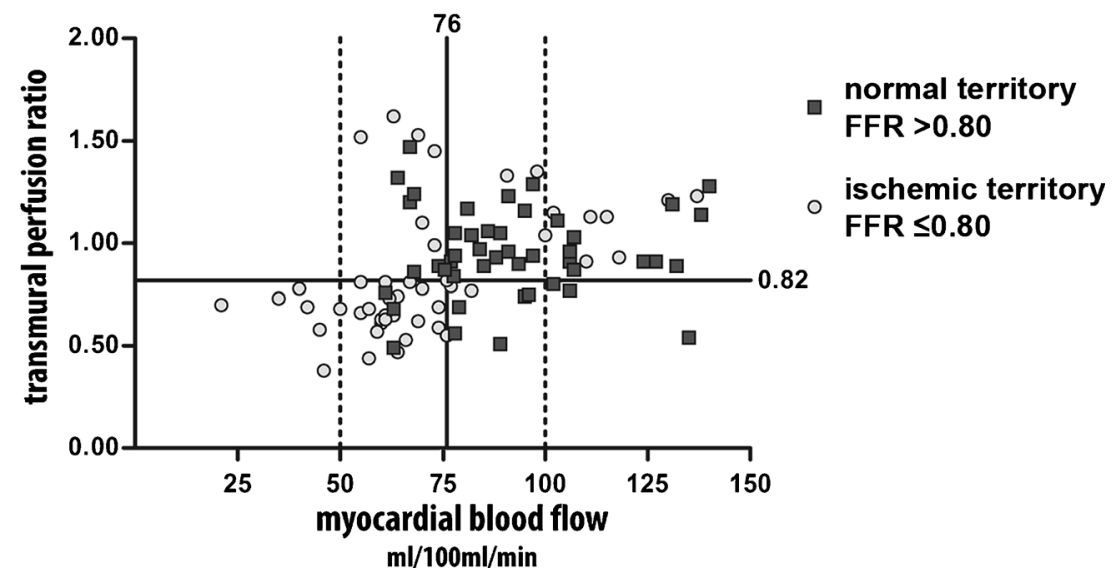

Fig. 5 Classification by MBF and TPR: Scatterplot showing the combined classification by MBF and TPR. The solid lines represent the diagnostic threshold for MBF (76) and TPR (0.82). A larger proportion of ischaemic territories was observed in the bottom left quarter, representing territories with a concordant abnormal MBF and TPR. The area between

the two vertical dashed lines represents the territories with an intermediate MBF between 50 and $100 \mathrm{ml} / 100 \mathrm{ml} / \mathrm{min}$. TPR: transmural perfusion ratio, $M B F$ : myocardial blood flow, FFR: fractional flow reserve 
hardening and partial volume effects potentially obscuring subtle perfusion defects. Future research related to improving MBF reconstruction in the endocardial layer adjacent to the ventricle cavity is of importance as the endocardial layer is more susceptible to myocardial ischaemia and perfusion imaging defects [29].

\section{Limitations}

These results are based on a limited number of patients recruited over a relatively long period of time (4 years) from a singlecentre study. As a result of the study complexity, as well as logistic factors such as availability of researchers and competing competitive research, only a fraction of the potentially eligible patients were recruited in this study. While the nonconsecutive enrolment was mostly based on these logistic factors, some degree of selection bias cannot be excluded. In a clinical setting CT-MPI will most likely be performed in conjunction with coronary CTA. However, this study focused on the diagnostic performance of CT-MPI specifically. As the diagnostic performance of dynamic CT-MPI using manual sampling of absolute MBF values is already good, a larger sample size might be needed to demonstrate an incremental value of other parameters. Motion correction algorithms were used if indicated; however especially around the edge of the MBF colour-coded images myocardium displacement artefacts can still be present. In several cases these artefacts result in high MBF values directly next to the left ventricle lumen. Even though care was taken to avoid these artefacts they may have negatively affected the performance of TPR. In this study preference was given to a robust, relatively user-independent transmural MBF profile curve as a basis for TPR. However, a more flexible freehand ROI in the endocardial and epicardial layer might affect TPR.

\section{Conclusion}

Transmural perfusion ratio measurements are feasible from dynamic CT-MPI and can identify functional obstructive CAD. The transmural perfusion ratio, as investigated in this study, from dynamic CT-MPI is inferior to and has limited incremental value on top of absolute myocardial blood flow measurements. In the future other myocardial flow parameters may be investigated to enhance the diagnostic performance of dynamic CT-MPI to identify myocardial ischaemia.

Acknowledgments The scientific guarantor of this publication is Koen Nieman. The authors of this manuscript declare relationships with the following companies: Marcel L. Dijkshoorn, Consultant, Siemens AG; Robert-Jan M. van Geuns, speaker's fee from Abbott Vascular; Koen Nieman, institutional research support from Bayer Healthcare, speaker's fee from GE Healthcare, Toshiba Medical Systems and Siemens Medical Solutions.
This study has received funding from The Dutch Heart Foundation. No complex statistical methods were necessary for this paper. Institutional Review Board approval was obtained. Written informed consent was obtained from all subjects (patients) in this study. Some study subjects or cohorts have been previously reported in Rossi A, Dharampal A, Wragg A, et al. (2014) Diagnostic performance of hyperaemic myocardial blood flow index obtained by dynamic computed tomography: does it predict functionally significant coronary lesions? Eur Heart $\mathrm{J}$ Cardiovasc Imaging, 15:85-94.

Methodology: prospective, diagnostic or prognostic study, performed at one institution.

Open Access This article is distributed under the terms of the Creative Commons Attribution 4.0 International License (http:// creativecommons.org/licenses/by/4.0/), which permits unrestricted use, distribution, and reproduction in any medium, provided you give appropriate credit to the original author(s) and the source, provide a link to the Creative Commons license, and indicate if changes were made.

\section{References}

1. Nagel E, Klein C, Paetsch I et al (2003) Magnetic resonance perfusion measurements for the noninvasive detection of coronary artery disease. Circulation 108:432-437

2. Uren NG, Melin JA, De Bruyne B, Wijns W, Baudhuin T, Camici PG (1994) Relation between myocardial blood flow and the severity of coronary-artery stenosis. N Engl J Med 330:1782-1788

3. Bamberg F, Klotz E, Flohr T et al (2010) Dynamic myocardial stress perfusion imaging using fast dual-source $\mathrm{CT}$ with alternating table positions: initial experience. Eur Radiol 20:1168-1173

4. Rossi A, Merkus D, Klotz E, Mollet N, de Feyter PJ, Krestin GP (2014) Stress myocardial perfusion: imaging with multidetector CT. Radiology 270:25-46

5. Bamberg F, Becker A, Schwarz F et al (2011) Detection of hemodynamically significant coronary artery stenosis: incremental diagnostic value of dynamic CT-based myocardial perfusion imaging. Radiology 260:689-698

6. Greif M, von Ziegler F, Bamberg F et al (2013) CT stress perfusion imaging for detection of haemodynamically relevant coronary stenosis as defined by FFR. Heart 99:1004-1011

7. Rossi A, Dharampal A, Wragg A et al (2014) Diagnostic performance of hyperaemic myocardial blood flow index obtained by dynamic computed tomography: does it predict functionally significant coronary lesions? Eur Heart J Cardiovasc Imaging 15:85-94

8. Ishida M, Kitagawa K, Ichihara $\mathrm{T}$ et al (2016) Underestimation of myocardial blood flow by dynamic perfusion CT: explanations by two-compartment model analysis and limited temporal sampling of dynamic CT. J Cardiovasc Comput Tomogr 10(3):207-214

9. Duncker DJ, Koller A, Merkus D, Canty JM Jr (2015) Regulation of coronary blood flow in health and ischemic heart disease. Prog Cardiovasc Dis 57:409-422

10. George RT, Arbab-Zadeh A, Miller JM et al (2009) Adenosine stress 64- and 256-row detector computed tomography angiography and perfusion imaging: a pilot study evaluating the transmural extent of perfusion abnormalities to predict atherosclerosis causing myocardial ischemia. Circ Cardiovasc Imaging 2:174-182

11. Flohr TG, Stierstorfer K, Ulzheimer S, Bruder H, Primak AN, McCollough $\mathrm{CH}$ (2005) Image reconstruction and image quality evaluation for a 64-slice CT scanner with z-flying focal spot. Med Phys 32:2536-2547

12. Niemann T, Henry S, Faivre JB et al (2013) Clinical evaluation of automatic tube voltage selection in chest CT angiography. Eur Radiol 23:2643-2651 
13. Mahnken AH, Klotz E, Pietsch H et al (2010) Quantitative whole heart stress perfusion $\mathrm{CT}$ imaging as noninvasive assessment of hemodynamics in coronary artery stenosis: preliminary animal experience. Investig Radiol 45:298-305

14. Mahnken AH, Bruners P, Katoh M, Wildberger JE, Gunther RW, Buecker A (2006) Dynamic multi-section CT imaging in acute myocardial infarction: preliminary animal experience. Eur Radiol 16:746-752

15. Schneider CA, Rasband WS, Eliceiri KW (2012) NIH Image to ImageJ: 25 years of image analysis. Nat Methods 9:671-675

16. Youden WJ (1950) Index for rating diagnostic tests. Cancer 3:32-35

17. Genders TS, Spronk S, Stijnen T, Steyerberg EW, Lesaffre E, Hunink MG (2012) Methods for calculating sensitivity and specificity of clustered data: a tutorial. Radiology 265:910-916

18. Bossuyt PM, Reitsma JB, Bruns DE et al (2003) The STARD statement for reporting studies of diagnostic accuracy: explanation and elaboration. Ann Intern Med 138:1-12

19. DeLong ER, DeLong DM, Clarke-Pearson DL (1988) Comparing the areas under two or more correlated receiver operating characteristic curves: a nonparametric approach. Biometrics 44:837-845

20. Bache RJ, Schwartz JS (1982) Effect of perfusion pressure distal to a coronary stenosis on transmural myocardial blood flow. Circulation 65:928-935

21. Barmeyer AA, Stork A, Muellerleile K et al (2007) Contrastenhanced cardiac MR imaging in the detection of reduced coronary flow velocity reserve. Radiology 243:377-385

22. Danad I, Raijmakers PG, Harms HJ et al (2014) Impact of anatomical and functional severity of coronary atherosclerotic plaques on the transmural perfusion gradient: a [15O]H2O PET study. Eur Heart J 35:2094-2105

23. Hosokawa K, Kurata A, Kido T et al (2011) Transmural perfusion gradient in adenosine triphosphate stress myocardial perfusion computed tomography. Circ J 75:1905-1912

24. Ko BS, Cameron JD, Leung M et al (2012) Combined CT coronary angiography and stress myocardial perfusion imaging for hemodynamically significant stenoses in patients with suspected coronary artery disease: a comparison with fractional flow reserve. JACC Cardiovase Imaging 5:1097-1111

25. Yang DH, Kim YH, Roh JH et al (2015) Stress myocardial perfusion CT in patients suspected of having coronary artery disease: visual and quantitative analysis-validation by using fractional flow reserve. Radiology 276:715-723

26. Huber AM, Leber V, Gramer BM et al (2013) Myocardium: dynamic versus single-shot CT perfusion imaging. Radiology 269:378-386

27. Kono AK, Coenen A, Lubbers M et al (2014) Relative myocardial blood flow by dynamic computed tomographic perfusion imaging predicts hemodynamic significance of coronary stenosis better than absolute blood flow. Investig Radiol 49:801-807

28. Wichmann JL, Meinel FG, Schoepf UJ et al (2015) Absolute versus relative myocardial blood flow by dynamic CT myocardial perfusion imaging in patients with anatomic coronary artery disease. AJR Am J Roentgenol 205:67-72

29. Bamberg F, Marcus RP, Becker A et al (2014) Dynamic myocardial CT perfusion imaging for evaluation of myocardial ischemia as determined by MR imaging. JACC Cardiovasc Imaging 7:267-277 